\title{
DIAGNOSTIC PARAMETERS DETERMINATION OF AIRCRAFT HYDRAULIC SYSTEM
}

\author{
Karel Třetina ${ }^{1}$, Tomáš Cäsar ${ }^{2}$ \\ ${ }^{1}$ Institute of Aerospace Engineering \\ Brno University of Technology \\ Technická 2, 61669 Brno, Czech Republic \\ tretina@fme.vutbr.cz \\ ${ }^{2}$ Institute of Aerospace Engineering \\ Brno University of Technology \\ Technická 2, 61669 Brno, Czech Republic \\ 152414@vutbr.cz
}

Keywords: hydraulic system, diagnostic symptom, technical condition, matrix of technical state, entropy, diagnostic parameter selection, information theory

\begin{abstract}
The article presents methodology for application of information theory for selection of hydraulic system diagnostic parameters. Changes in technical state of the system are accompanied by a set of symptoms. Method of determining information value of each symptom by information theory is presented. A set of technical conditions and set of symptoms for a simple hydraulic system are determined. Matrix of technical state describing relations between technical conditions and symptoms is constructed. The four symptoms are determined by matrix analysis with use of information theory. These symptoms are chosen as diagnostic parameters and provide the most information about technical state of the hydraulic system.
\end{abstract}

\section{INTRODUCTION}

The complexity of the aircraft hydraulic system with high standards of reliability and maintenance requires effective methods of examining their function and condition. During diagnosis is usually being looked for the answers to two basic questions [1]:

1. Is the controlled object without defect (operational) or it's faulty and can't work well?

2. What is causing faulty state of the system?

To answer these questions, two tasks need to be resolved:

1. to identify the minimum number of diagnostic, parameters, which will characterize the technical state of the system.

2. to determine the most advantageous sequence of parameter checks, in order to find defect, which caused the system failure.

Aim of this article is to answer the first of these two tasks. Because different changes in system parameters can indicate different failures, proper set of them characterizing unequivocally each of possible failures must be found. One of the ways of doing this is to quantify contribution of each symptom to the knowledge about the system, which can be done by use of information theory and then use ones, which give the most information. 
There are described analytical procedures of determining information value of measured parameters, which can then be used to find ones suitable for diagnostic parameters. The practical part is concerned with the use of this methodology for selecting minimum set of diagnostic parameters of small aeroplane hydraulic system.

\section{TECHNICAL STATE OF THE SYSTEM}

In the aeronautical diagnostics are distinguished only two states: operational and fault. Operational states means, that aircraft can fly, whereas fault state means that it cannot [2].

The number of states of the system, which consists of " $k$ " elements:

$S=2^{k}$

The number of failure modes is:

$S=2^{k}-1$

For example, when the system is composed of two elements, then:

- Both elements fully serviceable

- The first element is serviceable, the second is fault

- The first element is fault, the other is a serviceable

- Both elements are fault

With an increasing number of system elements, the number of states greatly increases. From a statistical analysis of failures of aircraft onboard systems generally indicate that the probability of two or more primary failures of individual elements of which the system is composed, is relatively very small. Therefore, in dealing with the inspection method can be assumed that the number of fault conditions the system is equal to the number of system elements. It is also possible to omit those states whose probability of formation during operation is very small.

\subsection{Symptoms of technical condition}

In medicine, doctor can use symptoms like increased temperature to diagnose angina. Similar methods can be applied on aircraft systems to identify the cause of a system failure.

Technical conditions and their symptoms may be related in the following ways [3]:

- one symptom characterizes clearly only one technical condition of the system

- one symptom is characteristic for several technical conditions of the system

- technical condition of the system can be uniquely characterized by only a few symptoms

The relationship between the technical state $S_{i}$ of the system and the individual symptoms $X_{j}$ can be determined by theoretical and analytical analysis or by experiments. For example, an active experiment (experimental simulation of fault conditions of the system).

Dependence of system status and corresponding symptoms can be displayed in tabular form by a matrix called state of the system. Each of the matrix columns corresponds to one of technical condition, each row represents one symptom. A simple form of record is that when a certain condition produces symptoms one is written to the corresponding location in a matrix, if not, zero is written. In this form, strength of each relation is not considered, thus all links are from a diagnostic point of view, of equal importance [4]. 
Assembling a matrix of system states based on analytical, experimental and of experience work is one of the main factors in finding the minimum set of diagnostic parameters and for determining an effective process of monitoring and evaluation of the technical condition of the hydraulic system of the aircraft.

After assembly of the matrix of system conditions for the hydraulic system, indeterminate interconnections between the states of the system and their symptoms can be expected from which must be select the ones that will become a set of diagnostic parameters. Purposeful seems therefore to determine the value of the information about the state of the system that carries each of the symptoms. When the value of information carried by each measurable symptom is known, appropriate set of diagnostic parameters can be selected.

\subsection{Quantitative valuation of information carried by symptoms}

To determine the quantitative value of information carried by each symptom, it is possible to use methods provided by information theory.

Theory of information characterizes the two-level system uncertainty, the entropy $H(S)$ of its condition which is defined as $[5,6]$

$$
H(S)=-\sum_{i=1}^{N} p_{i} \log p_{i}
$$

Where: $\quad N$ is the number of possible (expected) states of system

$p_{i}$ is the probability that the system will be in the i-th state

The numerical value of the entropy of such system can be determined according to the choice of basis in respect of the definition (1).

For systems that are characterized only by serviceable and fault mode is the base of the logarithm in the definition (1) set to 2 . Then unit entropy for $N=2$ and $p_{1}=p_{2}=0,5$ is

$$
H(S)=-\sum_{i=1}^{N} p_{i} \log _{2} p_{i}=-\left(\frac{1}{2} \log _{2} \frac{1}{2}+\frac{1}{2} \log _{2} \frac{1}{2}\right)=1
$$

Assuming that all possible (expected) states of the system will have the same probability of establishment, then $p_{i}=$ const $=\frac{1}{N}$. The total entropy of the considered systems has value

$$
H(S)=-\sum_{i=1}^{N} p_{i} \log _{2} p_{i}=-\sum_{i=1}^{N} \frac{1}{N} \log _{2} \frac{1}{N}=-N \frac{1}{N} \log _{2} \frac{1}{N}=\log _{2} N
$$

After measuring of one symptom, observed system will change its uncertainty. Controlled parameter carries a certain value of the information on the technical state of the system. The new value system uncertainty can be called conditional entropy and can be indicated $H\left(S / X_{j}\right)$. This value of uncertainty is likely to be lower than the original value $H(S)$, because after review of the parameter, there are some information on the technical state of the system already known.

The difference, between the initial value of entropy and its value after analyzing parameter $X_{j}$, is the state information system $I_{1}\left(X_{j}\right)$, which is given by symptom $X_{j}$. Therefore:

$$
I_{1}\left(X_{j}\right)=H(S)-H\left(S / X_{j}\right)
$$

The value of the conditional entropy $H\left(S / X_{j}\right)[5,6]$ is determined as the sum of the entropy of two parameters, the first of which includes $m$ the states to which monitored flag responds $X_{j}$ and the 
second one includes $n$ states to which the parameter does not respond. It is clear that with regard to prepared state of the system matrix, then the value $m$ is the number of ones and the number $n$ of zeros in the row, thus

$$
H\left(S / X_{j}\right)=\frac{m}{N} \log _{2} m+\frac{n}{N} \log _{2} n
$$

Value of the information, carried by the condition parameter, is

$$
I_{1}\left(X_{j}\right)=H(S)-H\left(S / X_{j}\right)=\log _{2} N-\left(\frac{m}{N} \log _{2} m+\frac{n}{N} \log _{2} n\right)
$$

Because we can write that $\log _{2} N=\frac{m}{N} \log _{2} N+\frac{n}{N} \log _{2} N$, the value of information carried by the parameter $X_{j}$ is

$$
I_{1}\left(X_{j}\right)=-\left(\frac{m}{N} \log _{2} \frac{m}{N}+\frac{n}{N} \log _{2} \frac{n}{N}\right)
$$

Said expression (7) for the value of the information carried by each parameter $X_{j}$ can be used to determine the loss of system entropy upon review of each of the parameters $X_{j}$.

\subsection{The minimum set of diagnostic parameters}

Parameters chosen as diagnostic have to give complete information on the technical condition of the system, thus sum of information value given by each of the parameters must equal initial system entropy.

$$
\sum I_{i}\left(X_{j}\right)=H(S)
$$

Of all the parameters that can be assessed by the state matrix, it is preferable to choose those that give the greatest value information at each successive step.

After determining the information assets of the individual measured parameters $X_{j}$ from the matrix of conditions and selecting an appropriate one to set of diagnostic parameters, it is necessary to rebuild the matrix condition so that in the next step of determining the information values of each parameter, the selected parameter had information value equal to zero.

In order to determine the value of information of all remaining parameters after the selected parameter is included in a set of diagnostic parameters and gave system status information, matrix is split to two parts, of which the first includes failures, to which the chosen parameter responds and the second to which the parameter does not respond. For thus rebuilt matrix, which is composed of two tables the value of the information is determined according to

$$
I_{2}\left(X_{j}\right)=-\sum_{1}^{r} \frac{m+n}{N}\left(\frac{m}{m+n} \log _{2} \frac{m}{m+n}+\frac{n}{m+n} \log _{2} \frac{n}{m+n}\right)
$$

After selection of a second suitable diagnostic parameter, the table adjustments continue under the same principle of separation of groups of parameters to subgroups for selected diagnostic parameter on the condition react and not react according to new selected parameter.

$$
I_{i}\left(X_{j}\right)=-\sum_{1}^{r} \frac{m+n}{N}\left(\frac{m}{m+n} \log _{2} \frac{m}{m+n}+\frac{n}{m+n} \log _{2} \frac{n}{m+n}\right)
$$


Group division and calculation of the information assets of the individual measured parameters continues, until at least one of the parameters gives some information about the technical state of the system or the entropy is reduced to zero.

\section{SELECTION OF DIAGNOSTIC PARAMETERS FOR THE HYDRAULIC SYSTEM OF FLAPS}

\subsection{Basic hydraulic system for control of flaps}

Figure 1 depicts basic hydraulic system used for control of flaps. Goal of this example is to choose diagnostic parameters out of nine offered, which can precisely identify cause of the failure from the eight possible given below $[1,7]$.

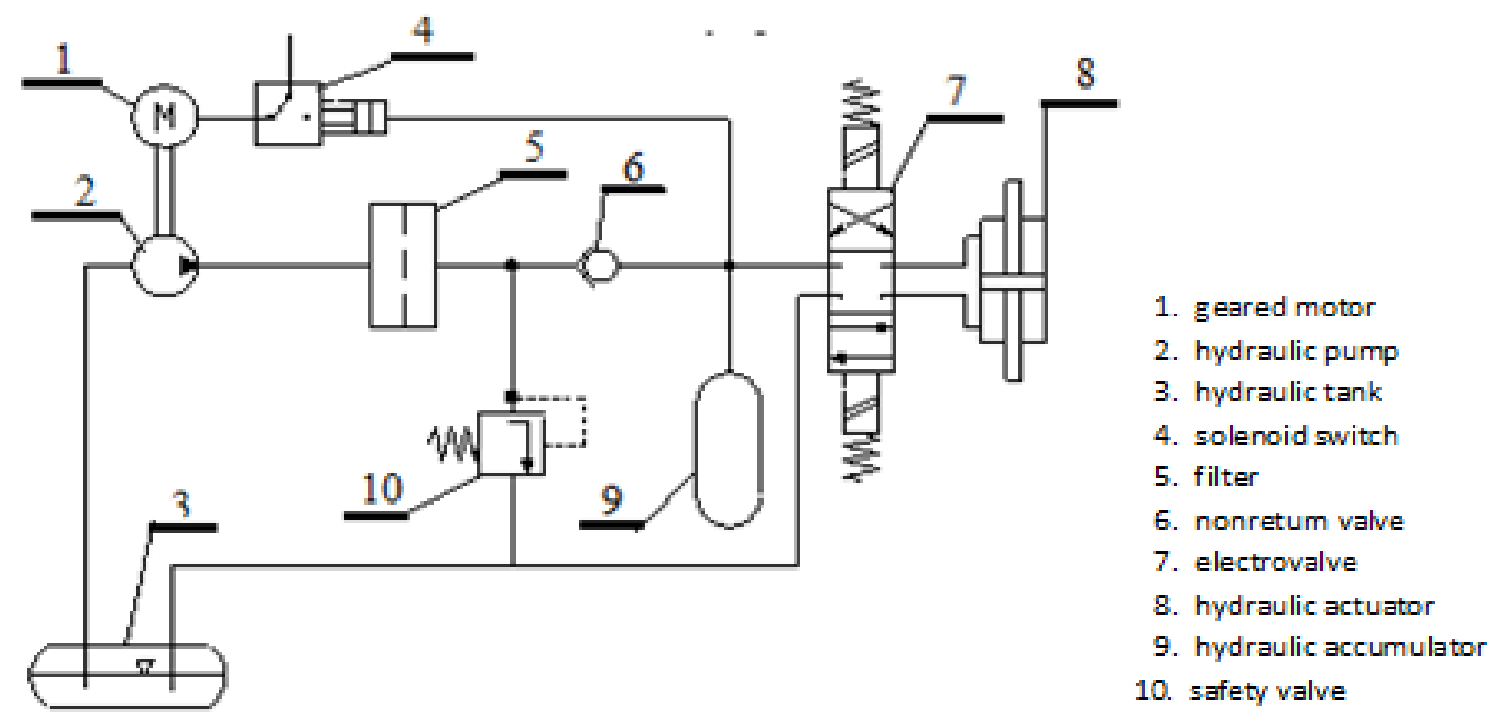

Figure 1: Hydraulic system used for control of flaps [4]

The set of technical conditions of hydraulic The set of parameter changes in technical states system $S_{i}$ (failures)

$X_{j}$ (symptoms)

1. Violation of pressure in the tank

1. The pressure drop across the filter

2. Enlarged pressure drop across the filter

2. Tribo of liquid

3. Wear of the pump

3. Pressure behind the pump (pulsation)

4. Reduced motor output

4. Pressure in front of accumulator

5. Leakage - violation of safety valve

5. Temperature behind hydro generator

6. Leakage of check valve

6. Pump speed

7. gas pressure drop in the ACU

7. Electric motor input current

8. Leakage of hydraulic motor

8. Time of piston displacement of actuator

9. Nitrogen pressure in the oil tank

Assuming equally probable states $p_{i}=1 / N=1 / 8$ the entropy of the system depends on the number of considered failures, the possible states of the system.

$$
H(S)=-\sum_{i=1}^{N} p_{i} \log p_{i}=-\sum_{1}^{8} \frac{1}{8} \log _{2} \frac{1}{8}=3
$$

This value of entropy, the uncertainty of the system will be reduced by checking the individual parameters.

\subsection{Matrix of the technical state of the system}


Table T1 represents the basic matrix of the technical state of the considered hydraulic system. The expected links between the technical conditions and their symptoms are listed there. In the last matrix column, state information values $I_{1}\left(X_{j}\right)$ are calculated, these individual information are calculated according to the equation:

$$
I_{1}\left(X_{j}\right)=-\left(\frac{m}{N} \log _{2} \frac{m}{N}+\frac{n}{N} \log _{2} \frac{n}{N}\right)=-\left(\frac{m}{8} \log _{2} \frac{m}{8}+\frac{n}{8} \log _{2} \frac{n}{8}\right)
$$

where $m$ is the number of one units and $n$ is the number of nulls, in each row.

\begin{tabular}{|c|c|c|c|c|c|c|c|c|c|}
\hline$S_{i} / X_{j}$ & 1 & 2 & 3 & 4 & 5 & 6 & 7 & 8 & $I_{I} X_{j}$ \\
\hline 1 & 1 & 1 & 1 & 1 & 1 & 0 & 1 & 1 & 0,544 \\
\hline 2 & 1 & 1 & 1 & 1 & 0 & 0 & 1 & 0 & 0,954 \\
\hline 3 & 0 & 0 & 1 & 1 & 0 & 1 & 1 & 0 & 1,000 \\
\hline 4 & 0 & 0 & 0 & 0 & 0 & 1 & 0 & 0 & 0,544 \\
\hline 5 & 0 & 1 & 1 & 0 & 1 & 0 & 1 & 0 & 1,000 \\
\hline 6 & 0 & 1 & 0 & 0 & 0 & 1 & 0 & 0 & 0,811 \\
\hline 7 & 0 & 1 & 1 & 1 & 0 & 0 & 1 & 0 & 1,000 \\
\hline 8 & 1 & 1 & 1 & 0 & 0 & 0 & 0 & 0 & 0,954 \\
\hline 9 & 0 & 1 & 1 & 0 & 0 & 0 & 1 & 0 & 0,954 \\
\hline$r$ & \multicolumn{7}{|c|}{1} \\
\hline
\end{tabular}

Table 1: Matrix of technical state 1

\begin{tabular}{|c|c|c|c|c|c|c|c|c|c|}
\hline$S_{i} / X_{j}$ & 3 & 4 & 6 & 7 & 1 & 2 & 5 & 8 & $I_{2} X_{j}$ \\
\hline 1 & 1 & 1 & 0 & 1 & 1 & 1 & 1 & 1 & 0,425 \\
\hline 2 & 1 & 1 & 0 & 1 & 1 & 1 & 0 & 0 & 0,925 \\
\hline 3 & 1 & 1 & 1 & 1 & 0 & 0 & 0 & 0 & 0,0 \\
\hline 4 & 0 & 0 & 1 & 0 & 0 & 0 & 0 & 0 & 0,425 \\
\hline 5 & 1 & 0 & 0 & 1 & 0 & 1 & 1 & 0 & 1,000 \\
\hline 6 & 0 & 0 & 1 & 0 & 0 & 1 & 0 & 0 & 0,849 \\
\hline 7 & 1 & 1 & 0 & 1 & 0 & 1 & 0 & 0 & 0,849 \\
\hline 8 & 1 & 0 & 0 & 0 & 1 & 1 & 0 & 0 & 0,925 \\
\hline 9 & 1 & 0 & 0 & 1 & 0 & 1 & 0 & 0 & 0,925 \\
\hline$r$ & \multicolumn{7}{|c|}{1} & \multicolumn{7}{|c|}{2} & \\
\hline
\end{tabular}

Table 2: Matrix of technical state 2

It follows from the above calculation that the largest amount of information is carried by the parameters 3 - the pressure behind the hydro pump, 5 - the temperature behind the hydro pump and 7 - the magnitude of the current to the electric motor, or the input power to the system. From table 1 , it is possible to select one of the three parameters and consider it a diagnostic parameter. When selecting diagnostic parameter out of multiple ones with same value of carried information, simplicity of its registration should be considered, thus, the parameter 3 - the pressure behind the hydro pump and its course during the operation of the system - is a preferred parameter.

By selecting the diagnostic parameter from table 1, system information is obtained, system entropy decreases by value $I_{1}\left(X_{3}\right)=1$. To select another parameter, it is necessary to divide the state matrix and adjust it so that the further calculation of the information values of the individual parameters is such that the information carried by the selected parameter $X_{3}$ was zero. The matrix was split to two parts, one part contains states, to which parameter 3 responds, the other one to which it does not respond. This requirement corresponds to table 2 , which is used to calculate updated information values according to the equation (10).

The highest value of the information carries the parameter 5 - the liquid temperature behind the hydro pump. This parameter can be included in a set of diagnostic parameters. The value of the information that the technical state of the system carries is $I_{2}\left(X_{5}\right)=1$.

Entropy of the system decreased by measuring parameters 3 - pressure behind the hydro pump and 5 - temperature of the liquid at the output from the hydro pump.

To select another diagnostic parameter, it is necessary to divide the state matrix again, rearrange, and calculate the values of the information. Groups created in step one were split to subgroups according to reactions to parameter 2 , of which first contains ones and other contains to form a table number 3 . 
Table 3 shows that the largest information value have the parameters $2-$ tribo of liquid and 8 - the displacement time of the piston rod of the hydro actuator. After selecting parameter 2 - tribo of liquid as another diagnostic parameter, the status matrix is further adjusted by methods used in first two steps to the table 4.

\begin{tabular}{|c|c|c|c|c|c|c|c|c|c|}
\hline$S_{i} / X_{j}$ & 3 & 7 & 4 & 6 & 2 & 5 & 1 & 8 & $I_{3} X_{j}$ \\
\hline 1 & 1 & 1 & 1 & 0 & 1 & 1 & 1 & 1 & 0,250 \\
\hline 2 & 1 & 1 & 1 & 0 & 1 & 0 & 1 & 0 & 0,750 \\
\hline 3 & 1 & 1 & 1 & 1 & 0 & 0 & 0 & 0 & 0 \\
\hline 4 & 0 & 0 & 0 & 1 & 0 & 0 & 0 & 0 & 0,250 \\
\hline 5 & 1 & 1 & 0 & 0 & 1 & 1 & 0 & 0 & 0,0 \\
\hline 6 & 0 & 0 & 0 & 1 & 1 & 0 & 0 & 0 & 0,500 \\
\hline 7 & 1 & 1 & 1 & 0 & 1 & 0 & 0 & 0 & 0,500 \\
\hline 8 & 1 & 0 & 0 & 0 & 1 & 0 & 1 & 0 & 0,750 \\
\hline 9 & 1 & 1 & 0 & 0 & 1 & 0 & 0 & 0 & 0,250 \\
\hline$r$ & \multicolumn{1}{|c|}{1} & 2 & \multicolumn{3}{|c|}{3} & 4 & \multicolumn{3}{|c|}{} \\
\hline
\end{tabular}

Table 3: Matrix of technical state 3

\begin{tabular}{|c|c|c|c|c|c|c|c|c|c|}
\hline$S_{i} / X_{j}$ & 3 & 7 & 4 & 6 & 2 & 5 & 1 & 8 & $I_{4} X_{j}$ \\
\hline 1 & 1 & 1 & 1 & 0 & 1 & 1 & 1 & 1 & 0,0 \\
\hline 2 & 1 & 1 & 1 & 0 & 1 & 0 & 1 & 0 & 0,0 \\
\hline 3 & 1 & 1 & 1 & 1 & 0 & 0 & 0 & 0 & 0,0 \\
\hline 4 & 0 & 0 & 0 & 1 & 0 & 0 & 0 & 0 & 0,0 \\
\hline 5 & 1 & 1 & 0 & 0 & 1 & 1 & 0 & 0 & 0,0 \\
\hline 6 & 0 & 0 & 0 & 1 & 1 & 0 & 0 & 0 & 0,0 \\
\hline 7 & 1 & 1 & 1 & 0 & 1 & 0 & 0 & 0 & 0,0 \\
\hline 8 & 1 & 0 & 0 & 0 & 1 & 0 & 1 & 0 & 0,250 \\
\hline 9 & 1 & 1 & 0 & 0 & 1 & 0 & 0 & 0 & 0,0 \\
\hline$r$ & 1 & 2 & 3 & 4 & 5 & 6 & 7 & \\
\hline
\end{tabular}

Table 4: Matrix of technical state 4

Table 4 gives only one option to select, which is 8 - time of the displacement of the piston rod in the set of diagnostic parameters.

\section{SUMARY}

The following procedure for selecting diagnostic parameters according to the values of the information given on the technical state of the considered hydraulic system for controlling airplane flaps has been chosen as follows, which form a set of diagnostic parameters:

$X_{3} \quad$ Hydro pump pressure (pulsation)

$X_{5} \quad$ Temperature behind the hydro pump

$X_{2} \quad$ Tribo of liquid

$X_{8} \quad$ Time of displacement of the piston rod of the hydraulic motor

Diagnostic parameters $X_{2}, X_{3}, X_{5}, X_{8}$ give full information about the set of technical state of the considered system. The total value of the information that these four parameters hold about the system is

$$
\sum_{1}^{4} I_{i}\left(X_{j}\right)=I_{1}\left(X_{3}\right)+I_{2}\left(X_{5}\right)+I_{3}\left(X_{2}\right)+I_{4}\left(X_{8}\right)=1+1+0,75+0,25=3=H(S)
$$

This value is equal to previously computed system entropy, which means that these parameters give complete information about system state.

\section{CONCLUSION}

In this article, information theory presented in the beginning was used to find value of information provided by different parameters of hydraulic system. According to these values, four parameters were chosen as diagnostic. By observing and analyzing these four diagnostic parameters, it is possible to determine the technical state of the considered system, to determine unequivocally in which state from the set of technical states the monitored system is. This method proved efficient in determining minimum set of diagnostic parameters. 


\section{ACKNOWLEDGEMENT}

Authors gratefully acknowledge financial support provided by the ESIF, EU Operational Programme Research, Development and Education within the research project Center of Advanced Aerospace Technology (Reg. No.: CZ.02.1.01/0.0/0.0/16_019/0000826) at the Faculty of Mechanical Engineering, Brno University of Technology.

\section{REFERENCES}

[1] KOPÁČEK, J. (1990) Technická diagnostika hydraulických mechanismů. SNTL Praha.

[2] KRÁL, M., PAŘíZEK, J., TŘETINA, K.( 1985) Kontrola technického stavu LT. VA, Brno.

[3] KADRY, S. (2012) Diagnostics and Prognostics of Engineering Systems: Methods and Techniques. IGI Global.

[4] TŘETINA, K. (2006) Vztahy mezi technickým stavem a př́znaky v HS letadel. LÚ FSI, VUT Brno.

[5] HUTCHISON, D., KANADE, T., KITTLER, J. (2013) Information Theory, Combinatorics, and Search Theory: In Memory of Rudolf Ahlswede. Springer Berlin Heidelberg.

[6] PIERCE, J. R. (2012) An Introduction to Information Theory: Symbols, Signals and Noise. Dover Publications.

[7] MARE, J.-C. (2016) Aerospace Actuators 1: Needs, Reliability and Hydraulic Power Solutions. ISTE.

\section{COPYRIGHT STATEMENT}

The authors confirm that they, and/or their company or organization, hold copyright on all of the original material included in this paper. The authors also confirm that they have obtained permission, from the copyright holder of any third party material included in this paper, to publish it as part of their paper. The authors confirm that they give permission, or have obtained permission from the copyright holder of this paper, for the publication and distribution of this paper as part of the READ 2018 proceedings.

This is an open access article distributed under the Creative Commons Attribution License which permits unrestricted use, distribution, and reproduction in any medium, provided the original work is properly cited. (CC BY 4.0). 